\title{
Power control in cognitive radios, Internet-of Things (IoT) for factories and industrial automation
}

\author{
Ifiok E Etim, Jaswinder Lota, FHEA, SMIEEE \\ School of Architecture, Computing \& Engineering \\ University of East London, London United Kingdom
}

\begin{abstract}
Cognitive radio (CR) is fast emerging as a promising technology that can meet the machine-to machine (M2M) communication requirements for spectrum utilization and power control for large number of machines/devices expected to be connected to the Internet-of Things (IoT). Power control in CR as a secondary user can been modelled as a non-cooperative game cost function to quantify and reduce its effects of interference while occupying the same spectrum as primary user without adversely affecting the required quality of service (QoS) in the network. In this paper a power loss exponent that factors in diverse operating environments for IoT is employed in the non-cooperative game cost function to quantify the required power of transmission in the network. The approach would enable various CRs to transmit with lesser power thereby saving battery consumption or increasing the number of secondary users thereby optimizing the network resources efficiently.
\end{abstract}

Keywords-Cognitive radio, M2M, IoT, non-cooperative game, cost function, power exponent

\section{Introduction}

Internet technology originally based on human-to human (H2H) communication is now seeing a paradigm shift with inclusion of objects as machines or devices that can sense and communicate with each other. With such devices expected to surpass 50 billion by 2020 [1], machine-to machine (M2M) communication is expected to be a key element in future networks. Internet-of Things (IoT) would be able to connect these different devices offering a whole range of new services. Therefore a future network has to cater for various M2M requirements such as spectrum, power and cost. With a huge volume of devices anticipated to be connected, spectrum scarcity is a constraint that will influence the quality-of service (QoS). Small cell design, interconnection of cellular network to other wireless networks and cognitive radio (CR) are some of the solutions that can address this problem [1]. Since CR offers a solution to increase the spectrum efficiency by allowing secondary users (SU) to access licensed spectrum as long it does not disadvantage or cause interference to degrade the required quality of service $(\mathrm{QoS})$ of the primary user (PU) and can also be deployed across various spectral bands, it is increasingly seen as a viable solution for M2M in IoT [2]-[5].
However as such opportunistic spectral access by CRs is likely to introduce some level of interference to the PUs and lower the QoS, therefore one of the main functions of a CR network is to provide transmission opportunities for SUs, lowering interference to the PUs and simultaneously ensuring an optimal QoS for all users. Maintaining adequate power control is therefore crucial for realizing a CR network.

Game theory with its ability to solve complex and distributed problems is a novel approach wherein power control in CR network is modelled as a non-cooperative game [6]-[8]. In [1] a power control algorithm is proposed wherein the noncooperative game cost function is dependent on the distances of the PUs and SUs to the Cognitive Base Station (CBS). Another solution based on game theory is given in [10] which quantifies the signal-to-interference noise ratio (SINR) based on an iterative algorithm for 3G CDMA networks. In [11] the method is improved further for the Nash Equilibrium point i.e., when all the transmission power within the CR network reaches a steady equilibrium for fixed SINR, by incorporating an interference mitigation algorithm. In [12] an improved iterative algorithm is proposed where addition of a chaos function leads to faster convergence for which comparisons with other iterative models is given. Power control can be modelled as a non-cooperative game, to adaptively adjust the interference by increasing the penalty punishment in a cost function for the $i^{\text {th }}$ SU [13]. As the deployment of IoT would be across heterogeneous environments e.g. factories, inaccessible and confined areas such as for structural monitoring, free and urban spaces this opens up various challenges for CR deployment. Diverse operating environments affect the wireless signal propagation which vary power received due to the isotropic power loss, reflection, refraction, diffraction and scattering. The combined effects of which are quantified as the power loss exponent $n$. Presently to our best knowledge, no study has investigated power control in CR in these diverse environments and the power loss exponent is normally taken as a fixed value of 4 assuming as the worst case in the cost function [9]-[13]. This may lead to suboptimal solution as path losses in free space would be quite different as compared to factories and automation environments. Path loss exponent is a measure of how fast the signal attenuates as a function of distance, is different for these environments in which the CR network 
would be deployed for which the values are given in Table 1 [14]. This paper investigates power control in CR within these different environments and focuses in the underlay scenario where the PUs and SUs transmit simultaneously in the network. The cost function in [13] is employed to analyze the power control of $\mathrm{CR}$ in these environments.

\begin{tabular}{|l|c|c|}
\hline \multicolumn{1}{|c|}{ Environment } & $\begin{array}{c}\text { Path Loss Exponent, } \\
n\end{array}$ & $\begin{array}{c}\text { Used path loss } \\
\text { exponent }\end{array}$ \\
\hline $\begin{array}{l}\text { In building, Line of } \\
\text { sight (LOS) }\end{array}$ & $1.6-1.8$ & 1.6 \\
\hline Free Space & 2 & 2 \\
\hline $\begin{array}{l}\text { Factory with } \\
\text { obstructions, } \\
\text { industrial automation }\end{array}$ & $2-3$ & 3 \\
\hline $\begin{array}{l}\text { Shadowed Urban } \\
\text { Cellular Radio }\end{array}$ & $3-5$ & 4 \\
\hline
\end{tabular}

Table 1. Path loss Exponents for different Environments [14].

The rest of this paper is organized as follows. In section II, the CR network model is presented. In section III, the power control in CR model based on non-cooperative game theory and its parameters are given. Section IV presents the numerical results followed by conclusions in section $\mathrm{V}$.

\section{CR Network Model}

The deployment of IoT across different environments require the use of adequate CBS with different ranges for transmission. These may include cognitive sensors [15] deployed within homes with range below $40 \mathrm{~m}$ to central hubs in factories, sensors for automation or sensors on streets and traffic lamps where the range could be $\sim 500 \mathrm{~m}$. In this research, we consider a single cell scenario where $N$ SUs with CR terminals are operating in a network with a CBS together with one PU along with its Primary Base station (PBS). Such as in case of large factories wherein the CRs are positioned at different distances from the CBS to cover operational distances of these environments. It is assumed that there is no channel gain between the SUs and the PBS but there is a gain from the PU to the CBS denoted by $h_{i}$ as shown in the Figure 1. The link gains from SUs to CBS are given by $h_{i},(i=1 \ldots \ldots N)$ and the link gains $g_{i}$ represents the gains from SU to PU since there is some level of interference from the SU to the PU. The same model is used to analyze the performance of the network across different operational environments based on Table 1. The link gains are related to the path distances from the CBS by [16]:

$$
h=A d^{-n}
$$

where $A$ is a constant gain factor and d is the distance from the CBS. If the power of the $i^{\text {th }} \mathrm{SU}$ is given by $p_{i}$ and the power of the PU is denoted by $p_{0}$, then SINR of the $i^{\text {th }} \mathrm{SU}$ is given by [13]:

$$
\gamma_{i}=\frac{p_{i} h_{i}}{\sum_{j=1, j \neq 1}^{N} p_{j} h_{j}+p_{0} h_{0}+\sigma^{2}}
$$

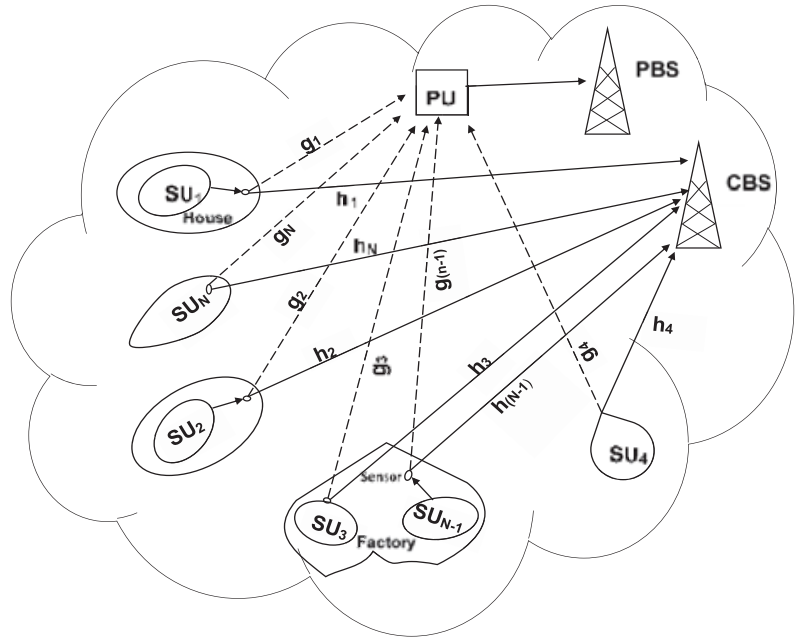

Fig.1 Model of the CR network

where $\sigma^{2}$ is an additive white Gaussian noise with zero mean and variance. To protect the SU and for a QoS, a SINR threshold is included such that:

$$
\gamma_{i} \geq \gamma_{i}^{t a r}
$$

In (3) $\gamma_{i}^{\text {tar }}$ is the SINR threshold for a QoS. As long as equation (3) is not violated, the system will guarantee the required QoS.

\section{Non-cooperative Game Power Control}

Game theory predicts the behaviors and choice of strategies of a group of players where every player has the goal of maximizing its own utilities. In a CR network the players are greedy and aim to increase power without consideration of other players so as to guarantee a QoS. This will increase the interference incurred by the PU thereby affecting its QoS. A non-cooperative game theory solves this problem by penalizing the contradicting CR thereby keeping them within acceptable limits. A non-cooperative game can be modelled in its basic form as [12]:

$$
G=[I, P, U]
$$

where the cognitive user combination is $I=\{1,2 \ldots \ldots N\}$, the strategy space is given by $P=p_{1} \times p_{2} \times \ldots \ldots \times p_{N}$ and utility cost function of the $i^{\text {th }} \mathrm{SU}$ is denoted as $\mathrm{U}_{\mathrm{i}}\left(p_{i}, p_{-i}\right)$. If $p_{-i}$ is the power of all other SUs except the $i^{t h} \mathrm{SU}$, the utility cost function is then given by [13]:

$$
u_{i}\left(p_{i}, p_{-i}\right)=a_{i} \log \left(\gamma_{i}-\gamma_{i}^{\text {tar }}\right)+b_{i} \sqrt{p_{i}^{\text {max }}-p_{i}}
$$

In (5) $a_{i}$ and $b_{i}$ are are constant non-negative weighting factors used in the adaptive cost (penalty) control for the $i^{\text {th }} \mathrm{SU}$ and $p_{i}^{\max }$ is the peak transmission power constraint of $i^{\text {th }} \mathrm{SU}$. The power and the power iteration are then given respectively as [13]: 


$$
\begin{aligned}
& f_{i}\left(p_{i}^{m}\right)=\frac{p_{i}^{(m)}}{\gamma_{i}^{(m)}} \gamma_{i}^{\text {tar }}+\frac{2 a_{i}}{b_{i}} \sqrt{p_{i}^{\max }-p_{i}^{(m)}} \\
& p_{i}^{(m+1)}=\left\{\begin{array}{c}
f_{i}\left(p_{i}^{m}\right), f_{i}\left(p_{i}^{m}\right)<p_{i}^{\max } \\
p_{i}^{\text {max }}, f_{i}\left(p_{i}^{m}\right) \geq p_{i}^{\text {max }}
\end{array}\right.
\end{aligned}
$$

The iterative power algorithm in (7) will continue to update till every strategy in the game is maximized. At this point, no individual can increase its utility by changing its strategy on its own and this is the solution to the non- cooperative game which is known as the Nash equilibrium (NE) point.

Definition 1: $(N E)$ : A power vector $\mathrm{S}=\left\{p_{1} \times p_{2} \times \ldots \ldots \times p_{N}\right\}$ is the NE of a game G. For $\forall_{I} \in N$ if and only if

$$
u_{i}\left(p_{i}^{*}, p_{-i}\right) \geq u_{i}\left(p_{i}, p_{-i}\right), \forall p_{i} \in s_{i}
$$

where $p_{-i}$ is the power of all SUs except $\mathrm{SU}_{\mathrm{i}}$ and is $p_{i}^{*}$ the NE solution of $\mathrm{SU}_{\mathrm{i}}$.

\section{Simulation and Analysis}

Simulations are taken in Matlab ${ }^{\circledR}$ R2015a for typical single cell network parameters. The IoT devices are placed with fixed distances of $20 \mathrm{~m}, 60 \mathrm{~m}, 90 \mathrm{~m}, 130 \mathrm{~m}, 190 \mathrm{~m}, 220 \mathrm{~m}, 290 \mathrm{~m}$ and $350 \mathrm{~m}$ from the CBS. This should effectively cover the operational distance of most CR deployed IoT devices such as wearable devices which should have range under $50 \mathrm{~m}$ to hand held two way radios in factories. The noise power variance $\sigma^{2}=$ $5 \times 10^{-15} \mathrm{~W}$. The PU transmission power $p_{o}=0.07 \mathrm{~W}$ and the SUs have an initial transmission power of $p_{i}=5 \times 10^{-10} \mathrm{~W}$. The maximum allowable transmission power of the $i^{\text {th }} \mathrm{CR}$ is $p_{i}^{\max }=$ $0.5 \mathrm{~W}$. The SINR threshold is specified as $\gamma^{\text {tar }}=7 \mathrm{~dB}$ and $2 \mathrm{a}_{\mathrm{i}} / \mathrm{b}_{\mathrm{i}}$ $=3 \times 10^{-4}$ as the initial value for iterations. The channel gains are given by (1), where $A=7.5 \times 10^{-3}$ and $n=[1.6,2,3,4]$ where each of the values represents the power exponents for various environments in Table 1. The transmit power of CR enabled IoT devices will be important for the success of such technology. This is because CR devices will seek to improve its own performance to optimal levels by increasing the transmitted power without which may interfere with other users. The average transmitted power of a CR is crucial to the QoS for both the PU and SU. If the average power is too high, it introduces interference into the network which in turn causes a degradation to the QoS of the PU, if the CR transmits low power, its own QoS may be affected if the signals have a lower signal-to-noise ratio (SNR) for the required bit-error rate (BER) resulting in dropped calls and or failed data transmission. As battery life for IoT devices is limited there is a need to ensure optimal transmission while preserving battery life in such devices. Figure 2 shows the average transmit power of the $i^{\text {th }} \mathrm{SU}$ as it varies with the environment within which the CR network is based.

The simulations shows different powers levels required for effective transmissions while operating in different environments. This is because power degradation due to propagation losses are accounted for in the power loss exponents which the transmitted power has to overcome. It can be deduced from the plots that the proper selection of the power loss exponent is crucial for adequate power transmission and effective battery life in IoT devices. The IoT device operating in line of sight (LOS) doesn't need to transmit with the power loss exponent for an urban cellular radio as this will result in higher transmitted than what is required thereby reducing battery life. IoT devices should be able to adaptively select the power loss exponent based on its operational environment during sensing or initial link-up. Within the right environment, the CR transmit power may be reduced without affecting the QoS or the available power may be used to accommodate more SUs without causing disruptive interference to the PUs.

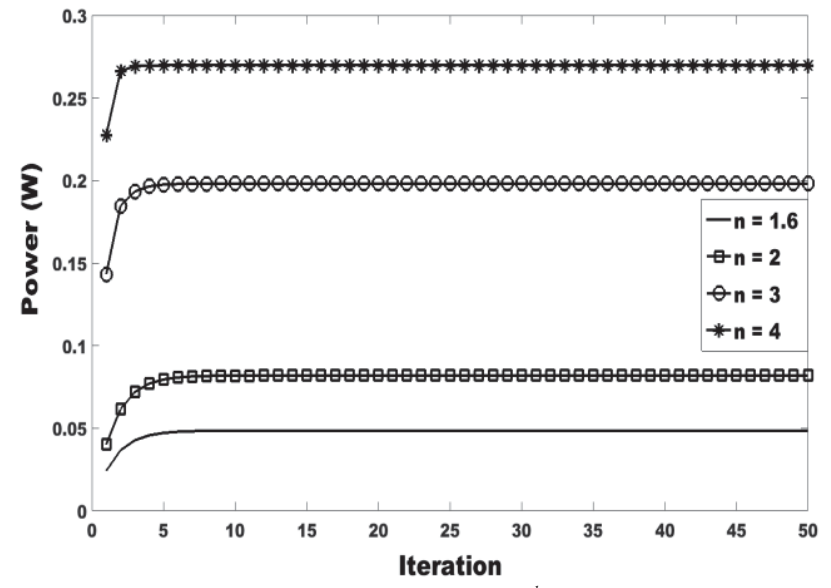

Fig. 2 Average Power required by the $i^{\text {th }}$ cognitive Radio

There is a risk that reducing transmit power may result in failed transmission as the IoT devices may not have sufficient power to reach the required SINR for effective transmission. In Figure 3, the average SINR of the SUs in the different environments are plotted. The plot shows that based on the fixed distance of the IoT devices in the network and for the correct path loss exponent, the performance of all the radios where all above the target SINR required to guarantee the QoS for both the PU and SU. This means that if the correct path loss exponent for the IoT device is selected, the non-cooperative game cost function via the iterative algorithm ensures the required QoS is met.

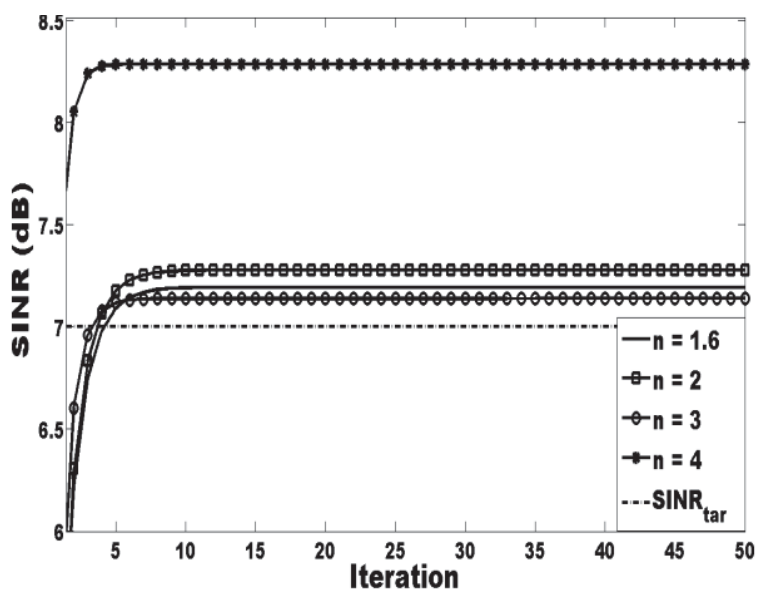

Fig. 3 Average SINR of the $i^{\text {th }} \mathrm{CR}$ 
Normally otherwise a single value of $n$ as 4 is chosen irrespective of the operating environment of the IoT devices. This does not always guarantee an improved performance of the CR IoT network. IoT devices will be deployed over different environments therefore the PU can be within any distance from the CBS. The effect of varying the PU distances over a range of $10 \mathrm{~m}-150 \mathrm{~m}$ on SINR is investigated for different environments and its variation is plotted in Figure 4. It is observed that the target SINR is achieved at distances of almost $30 \mathrm{~m}, 45 \mathrm{~m}, 70 \mathrm{~m}$ and $65 \mathrm{~m}$ for $n=1.6,2,3$ and 4 respectively. As the distance increases between the CBS and the PU, the user case of $n$ values crosses the target SINR to ensure acceptable QoS as seen on Figure 4. The increase in PU and CBS distances is because the IoT devices with higher $n$ transmit at higher powers as compared to lower values of $n$ as shown earlier in Figure 2 thereby increasing the PU distances for the target SINR. After the crossover the SINR increases at the fastest rate for $n=4$ than for others. In CR-IoT devices the PU distance affects the SINR and is crucial to selecting the correct path loss exponent specific to the environment. It gives an idea of the performance of the CR devices at various points and will help the device in the decision making of what environment in which it is deployed thereby always guaranteeing optimal SINR.

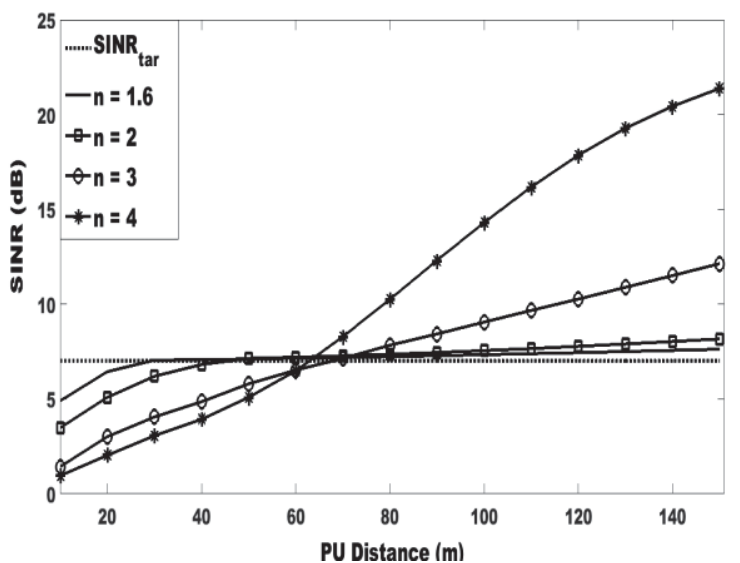

Fig. 4 Average SINR vs PU distances with fixed IoT device distances

In Figure 5, the variation in power required for optimal transmission by the $i^{\text {th }}$ IoT device at various distances is given. This shows the rate at which the transmit power of the $i^{\text {th }} \mathrm{CR}$ increases with respect to the distance of the $i^{\text {th }} \mathrm{SU}$ for each environment. It can be seen that the power required to transmit a signal increases at a faster rate as $n$ increases. This is to ensure that the signal reaches the CBS without failure to overcome the effects of higher propagation losses. If the SU continues to transmit assuming a path loss exponent of $n=4$ while being in the environment for $n=1.6$, though the QoS may be met for both PU and SU, it would transmitting at much higher power levels than required leading to excessive power usage and battery power drain.

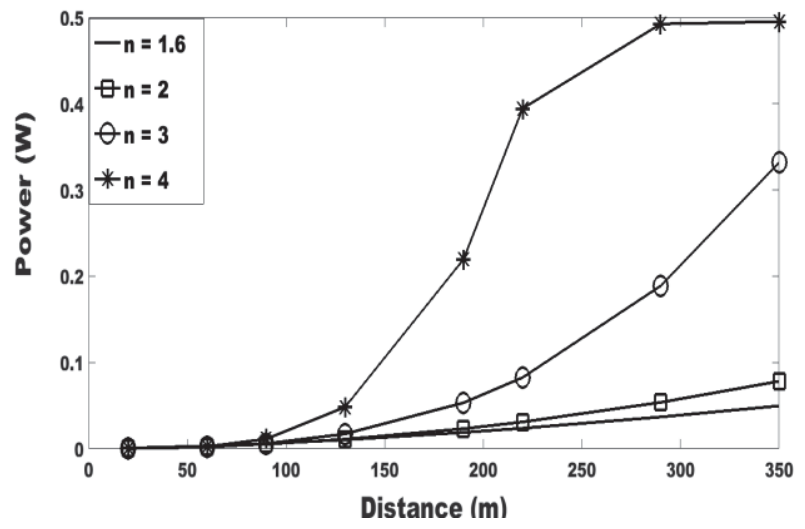

Fig. 5 Average Power of the $i^{\text {th }} \mathrm{CU}$ against Distance for each environment

\section{Conclusion}

Optimal power control in $\mathrm{M} 2 \mathrm{M}$ for $\mathrm{CR}$ deployment is critical to realize the anticipated services in IoT. For IoT the environment where the CR operates is important as different power levels are required to achieve effective transmission. A CR should therefore be able to detect the best channel properties so as to ensure optimal performance at all times thereby improving the use of the spectrum. Environments with higher propagation losses will require higher transmit power to ensure the required QoS than those with lower losses. These diverse environments that affect wireless propagation can be modelled into the non-cooperative game cost function with an accurate power loss exponent relevant for IoT deployment, which will reduce battery usage, and is critical for IoT services as these are likely to be deployed on low/ultra-low power sensor networks. Alternatively the $\mathrm{CR}$ network can accommodate more secondary users without lowering the QoS due to reduced interference. These results are based on Matlab simulations and the exact analysis for reduced power and increase in number of users is underway, the results of which would be reported in a future publication.

\section{References}

[1] A. Ali, W. Hamouda and M. Uysal, 'Next generation M2M cellular networks: challenges and practical considerations', IEEE Communications Magazine, vol. 53, no. 9, p. 18-24, Sept. 2015.

[2] A. Aijaz and A. Aghvami, 'Cognitive machine-to machine communications for Internet-of things: A protocol stack perspective', IEEE Internet of Things Journal, vol. 2, No. 2, p. 103-112, April 2015.

[3] R. Shigueta, M. Fonseca, A. Ziviani, AC Viana and A Munaretto 'A strategy for opportunistic cognitive channel allocation in wireless Internetof Things', IFIP Wireless Days (WD), Rio De Janeiro, Brazil, p. 1-3, Nov. 2014.

[4] E. Z. Tragos and V. Angelakis, 'Cognitive radio inspired M2M communications', 16th International Symposium on Wireless Personal Multimedia Communications (WPMC), Atlantic City, NJ, p. 1-5, June 2013.

[5] Y. H. Lin, Q. Wang, J. Wang, L. Shao and J. Tang, 'Wireless IoT platform based on SDR technology', IEEE International Conference on Green 
Computing and Communications, IEEE Internet of Things and IEEE Cyber Physical and Social Computing, Beijing China, p. 2245-2246, Aug. 2013.

[6] U. C. Saraydar, B. N. Mandayam and D. Goodman. 'Efficient power control via pricing in wireless data networks', IEEE Transactions on Communications, vol. 50, no. 2, p. 291 - 303, Feb. 2002.

[7] D. Goodman and N. Mandayam, 'Power control for wireless data'. IEEE Personal Communications, vol. 7, no. 2, p.48-54, April 2000.

[8] H. Shibiao, S. Zhiguang and A. Xiaodan, 'A novel non-cooperative power control game algorithm for cognitive radio' Telecommunication Engineering, vol. 48, no. 8, p.11-15, 2008.

[9] Y. Zu, Y. Liu, S. Mao and Y. Jia, 'Power Control Algorithm Based on SNR Cost Function in Cognitive Radio System'. IEEE 18th International Conference on Parallel and Distributed Systems (ICPADS), Singapore, p. 913-917, Dec. 2012.

[10]S. Koskie and Z. Gajic, 'A nash game algorithm for SIR-based power control in $3 \mathrm{G}$ wireless CDMA networks'. IEEE/ACM Transactions on Networking, vol. 13, no. 5, p. 1017-1026, Oct. 2005.

[11]L. Aik Jin and T. Chee Keong. 'A Nash-based power control game for green communications via cognitive radio networks'. IEEE Conference on Sustainable Utilization and Development in Engineering and Technology (STUDENT), Kuala Lumpur, p. 164-169, Oct. 2012.

[12]A. Al Talabani, A. Nallanathan, and H. X. Nguyen, 'A Novel Chaos Based Cost Function for Power Control of Cognitive Radio Networks'. IEEE Communications Letters, vol. 19, no. 4, p. 657-660, April 2015.

[13] Y. Guanglong, L. Bin, T. Xuezhi and W. Xiao, 'Adaptive power control algorithm in cognitive radio based on game theory'. IET Communications Letters, vol. 9, no. 15, p. 1807-1811, Oct. 2015.

[14] Miranda, J., et al. Path loss exponent analysis in Wireless Sensor Networks: Experimental evaluation. 11th IEEE International Conference on Industrial Informatics (INDIN), Bochum, Germany, July 2013.

[15] Vijay, G., E.B.A. Bdira, and M. Ibnkahla, Cognition in Wireless Sensor Networks: A Perspective. IEEE Sensors Journal. Vol. 11, No. 3, p. 582592, March 2011.

[16]Y. Zhu, J. Sun, S. Shao, L. Yang and H. Zhu. 'Nash Game-theoretical Algorithm for power control in cognitive radio networks' IEEE conference on Communication Technology, Nanjing, p. 730-733, Nov. 2010. 